\title{
Tuberculosis cases in cross-regional floating population from 2009 to 2017 in Shaanxi Province, China
}

Ke Men ( $\square$ menke@xiyi.edu.cn )

Xi'an Medical University https://orcid.org/0000-0001-5734-809X

Hairui Zhang

Xi'an Medical University

Weiping Liu

Shaanxi Provincial Institute for TB Control and Prevention

Henry Han

Fordham University

Research article

Keywords: Tuberculosis, Floating Population, Public Health

Posted Date: January 17th, 2020

DOI: https://doi.org/10.21203/rs.2.21199/v1

License: (a) (i) This work is licensed under a Creative Commons Attribution 4.0 International License.

Read Full License 
Tuberculosis cases in cross-regional floating population from 2009 to 2017 in Shaanxi Province, China

Ke Men ${ }^{1 *}$, Hairui Zhang ${ }^{1 *}$, Weiping Liu ${ }^{2}$, Henry Han $^{3}$

Correspondence to: Ke Men, Institute for Research on Health Information and Technology, School of Public Health, Xi'an Medical University, Xi'an, Shaanxi province, China. e-mail: menke@xiyi.edu.cn

${ }^{*}$ Contributed equally 


\begin{abstract}
Background: The cross-regional population of tuberculosis (TB) has become an important part of the prevention and control of TB cases in China with the gradually increasing of floating population. The aim of our study was to explore the main epidemiological characteristics and provide reference for the treatment and management of TB cases in cross-regional floating population in Shaanxi Province.
\end{abstract}

Methods: The data of TB cases in cross-regional floating population from 2009 to 2017 were derived from the National Tuberculosis Management Information Registration System. Descriptive analysis was applied for the baseline demographical, continuous data are expressed as means \pm standard deviation and categorical in percentage. The $\chi 2$ test, which was implemented as Fisher's exact test in this study, was applied to categorical variable comparisons.

Results: A total of 613 floating TB cases consisting of 413 males (67.4\%) and 200 females (32.6\%) were registered in Shaanxi province from 2009 to 2017. The difference of gender distribution was statistically significant $(P=0.039)$. Of the total, 190 cases (31.0\%) were smear-positive and 175 cases $(28.5 \%)$ were negative. From 2009 to 2017, the difference of the gender distribution was not statistically significant $(P=0.732)$, but the difference in the rate of transferred into original was found statistically significant $(P=0.000)$.

Conclusion: The number of TB cases in cross-regional floating population in Shaanxi Province showed a relatively stable trend from 2009 to 2017 . Young males should be given more attentions in TB control because they counted most of the TB cases.

Keywords: Tuberculosis, Floating Population, Public Health 


\section{Background}

Since Chinese government started the reform and opening-up policy in 1978, the floating population increased gradually over the past forty years. ${ }^{1}$ The floating population, mostly consisting of migrant workers, is made up of people who move to another area, but do not change their permanent residence. ${ }^{2}$ According to the National Bureau of Statistics of the People's Republic of China, there were 244 million floating population in 2017 , accounting for $17.5 \%$ of the total population. ${ }^{3}$ Most of the floating population live and work in poor conditions, have a low-income, and lack of nutrition and knowledge of health, which prevent them from obtaining enough social and health care resources. As a result, they are more likely to face risky health conditions than normal population. ${ }^{4}$

According to the Global Tuberculosis Report 2017 released by the World Health Organization (WHO), although the incidence of TB is slowly declining at a rate of $2 \%$ per year, there were still 10.4 million new cases and 1.7 million deaths worldwide in 2016. ${ }^{5}$ Seven countries, which include India, Indonesia, China, the Philippines, Pakistan, Nigeria, and South Africa, account for $64 \%$ of the global TB burden. ${ }^{5}$ Besides the rapid economy and society development, widespread and frequent population movements in China, drug resistance and poor control effect of TB have become an important cause of the clinical deterioration of tuberculosis. ${ }^{6}$

Cross-regional tuberculosis cases refer to those who have registered in the treatment process, and transferred from one county (district) to another, but cannot continue to receive treatment or management in the original hospital. ${ }^{7}$ The continuous movement of floating population of TB cases, who move back and forth between their homes and workplaces, posed a great difficulty in treating TB. ${ }^{8,9}$ As noted above, cross-regional population of TB has become an important part of the prevention and control of TB in China.

The current study aims at analyzing the epidemiological characteristics of cross- 
regional TB cases in Shaanxi Province from 2009 to 2017 and provides a theoretical foundation for the promotion and treatment management for the cross-regional floating population TB cases.

\section{Methods}

\section{Data collection}

The data of TB cases in cross-regional floating population in Shaanxi Province from 2009 to 2017 were retrieved from the National Tuberculosis Management Information Registration System. For each transferred patient, demographical data (such as age, gender, Ethnic group and occupation), types of diagnosis, types of treatments, and migration information were all collected from the system.

\section{Related indicators}

Rate of transferred in place $^{10}$ is the percent ratio between the actual number of TB transferred patients and the total number of TB registered transferred patients. The closer the ratio is to one hundred percent, the better the management effectiveness of TB registered transferred patients.

Cross-regional tuberculosis cases refer to the patients registered in a TB treatment process, transferred from one county (district) to another, but cannot continue to receive treatment or management in the original hospital. ${ }^{7}$

\section{Statistical analysis}

Data analysis was conducted by employing SPSS 20. Descriptive analysis was applied for the baseline demographical, continuous data are expressed as means \pm standard deviation and categorical in percentage. The $\chi^{2}$ test, which was implemented as Fisher's exact test in this study, was applied to categorical variable comparisons. The cut-off of p-value is set as 0.05 to determine statistical significance.

\section{Ethical considerations}

The principles of the Helsinki Declaration were considered. Written, informed consent 
was obtained from all study subjects. The study was approved by the medical ethical committee of Xi'an medical university.

\section{Results}

A total of 613 tuberculosis patients that consisted of 413 males (67.4\%) and 200 females $(32.6 \%)$ were registered in our study. The age of the patients follows a normal distribution with mean 34.21 years old and standard deviation (SD) 15.75 years old. In occupation status, $138(22.5 \%)$ cases were farmers, the largest group among all patients. 599(97.7\%) cases were Han ethnic group (Table 1).

From 2009 to 2017, the number of TB cases in cross-regional floating population demonstrated a relatively fluctuant trend of highest number 106 in 2010 and lowest number 50 in 2014(Figure 1). Our study indicated that most TB cases were young males. It is probably because young males are the majority in the cross-regional floating population. Our hypothesis test showed that gender difference was not statistically significant from 2009 to 2017 ( $P=0.732$; Table 2). But among different age groups, the gender difference was statistically significant $(P=0.039$; Table 3$)$.

The inspection and treatment situation were provided by Table 4 . The sputum smear inspection indicated that 190 cases $(31.0 \%)$ are positive and 75 cases $(28.5 \%)$ are negative. Of the total, 593 cases $(96.7 \%)$ were new cases and received the first treatment. During the registration distribution, in addition to 593 new cases, $16(2.6 \%)$ were retreatment cases and $4(0.7 \%)$ cases were failed in initial treatment.

Table 5 provided the management-situation of tuberculosis: there were 476 cases (79.7\%) transferred in place, but 116 cases not, besides 21 cases no feedback from 2009 to 2017. By the method of Fisher's exact test, there was a statistically significant difference in the rate of transferred in place from 2009 to $2017(P<0.01)$. Except of highest proportion 46(92.0\%) in 2014 and lowest proportion 56(65.1\%) in 2009, the annual rate of transferred in place presents a relatively stable trend. 


\section{Discussion}

Despite that the incidence rate of TB declined gradually in recent years, we still found that there were many people identified as new cases or even death because of TB. It was especially true for those patients from the floating population. It seemed to appear an increasing trend by years for the patients. ${ }^{3}$ According to national population census data, the number of floating population in Shaanxi province was increasing and the proportion of floating population to the resident population was growing evidently $(3.09 \%$ in $1990,6.69 \%$ in $2000,15.79 \%$ in 2000$) .^{31}$

From 2009 to 2017, there were 613 tuberculosis cases among the cross-regional floating population in Shaanxi province, the number of TB cases in cross-regional floating population presented a relatively stable trend, especially because the newly registered cases in 2017 were only 1.07 times of 2009 . On one hand, it shows that the prevalence of tuberculosis among the floating population in Shaanxi province has increased in recent years. On the other hand, with the increase in the number of registered cases, the newly discovered cases among the floating population has a higher rate of registration of cases, which is conducive to the management and control of tuberculosis diseases. ${ }^{10,12,13}$

As for the relationship between TB and gender, our study found that most of the TB cases were men. Such an observation is consistent with the previous TB studies in Beijing, ${ }^{14}$ Shanghai, ${ }^{15}$ Guangzhou, ${ }^{16}$ Shenzhen,${ }^{17}$ and Jiangsu. ${ }^{12}$ This is probably related to the rapid urbanization in Shaanxi province, where men are the majority in the floating population besides the major workforce. High labor intensity, long working hours and poor living conditions have led to a decline in personal physique and immunity, which has increased the chances of infection among this group. ${ }^{18}$ Some studies suggested that smoking and exposure to the dust or harmful chemical aerosols in working environments were risk factors for tuberculosis case. Furthermore, males were more likely to exposure to such risk factors in their lives or workplace compared to the females. ${ }^{19}$ 
In terms of the age, our study found that nearly $50 \%$ of the tuberculosis cases were aged between 20 and 30 years old. This is consistent with the findings from Africa ${ }^{20}$ and Dongguan. ${ }^{21}$ Such a result seems to indicate that tuberculosis mainly affects adolescents and young adults. This is probably because the new generation of migrant workers in the current China are young people born after 1980s. ${ }^{3}$ In addition, by occupation, farmers accounted for the highest proportion of cases in our study. Most of the floating populations are rural-to-urban migrant workers and they are identified as farmers instead of others in their health records. These people generally have long working hours, low income levels, poor living conditions and low healthcare awareness. These factors will result in a decline in personal physique and immunity for them. ${ }^{22}$

Early detection, regulation of treatment and management of tuberculosis patients are the key to controlling the spread of tuberculosis, and can also effectively prevent the emergence of drug-resistant tuberculosis. ${ }^{23}$ With the expansion of directly observed treatment and short course chemotherapy (DOTs) during tuberculosis control strategy in recent years, ${ }^{24}$ China has paid more and more attention to the tuberculosis cases of the floating population and integrated the prevention and treatment of tuberculosis into the comprehensive management of community health. The total transferred in place rate in Shaanxi province was $79.7 \%$, which is Higher than Hubei, ${ }^{25}$ Shenzhen, ${ }^{11}$ but slightly lower than Fujian. ${ }^{26}$ Our study indicates that after the initialization of the National Cross-regional Program for the Management of Tuberculosis Patients, the health administrative department taking migrant population as one of the key populations for tuberculosis prevention and treatment and the extensive work in combating tuberculosis has proved to be effective. Through health administrative intervention, medical institutions and prevention agencies to improve the medical service level of tuberculosis patients in floating population, free screening for the close contact with active tuberculosis, the use of special rapid information system for the management and comprehensive methods can be achieved the rate of transferred in place increasing year by year. ${ }^{27}$ 
Taken together, China is one of the high tuberculosis burden countries all over the world. Due to the high mobility of the floating population and the lack of a fixed residence, it is difficult to adhere to the prescribed course of treatment and easy to interrupt the treatment. ${ }^{28}$ Therefore, there are still certain challenges in the management of tuberculosis among the across-regions floating population, the floating population is still the key population for the future prevention and treatment of tuberculosis in Shaanxi Province. We must realize that it is important to create a convenient diagnosis and treatment environment for tuberculosis case in floating population, strive to improve patient compliance and the rate of success follow-up. It is necessary to give the floating population more human care, improve their working and living condition and the quality of life and further enhance the implementation of cross-regional tuberculosis case management procedures in reducing the occurrence and spread of tuberculosis in floating population. ${ }^{29,30}$

\section{Conclusion}

The number of TB cases in cross-regional floating population in Shaanxi Province showed a relatively stable trend from 2009 to 2017 . Young males should be given more attentions in TB control because they counted most of the TB cases.

\section{Abbreviations}

TB: tuberculosis; DOTs: directly observed treatment and short course chemotherapy

\section{Acknowledgment}

None

\section{Declarations}

None 
$\mathrm{KM}$ and $\mathrm{HZ}$ conceived the study, performed statistical analysis and drafted the manuscript. WL helped to collect the data. participated in the conception and design of the study. HH revised the manuscript. All authors read and approved the final manuscript.

\section{Funding}

This work was supported by National Natural Science Foundation of China, Grant No. 81773496.

\section{Availability of data and materials}

The datasets analyzed during the current study are not publicly available due to protection of the patients' privacy, but are available from the corresponding author on reasonable request.

\section{Ethics approval and consent to participate}

The principles of the Helsinki Declaration were considered. Written, informed consent was obtained from all study subjects. The study was approved by the medical ethical committee of Xi'an medical university.

\section{Consent for publication}

Not applicable.

\section{Competing interests}

The authors declare that they have no competing interests.

\section{Author details}

${ }^{1}$ Institute for Research on Health Information and Technology, School of Public Health, Xi'an Medical University, Xi'an, Shaanxi province, China

${ }^{2}$ Shaanxi Provincial Institute for TB Control and Prevention, Xi'an, Shaanxi province, China

${ }^{3}$ Department of Computer and Information Science, Fordham University, Lincoln 


\section{Center, New York, NY 10023, USA}

\section{References}

1 Migrant population Service Center of National Health and Family Planning Commission of PRC: China migrant survey. http://www.chinadrk.org.cn/wjw/\#/data/classify/population/yearList. Accessed July 2018.

2 Yang J H. Research on social integration of China's floating population. Social Sciences in China 2015; 2: 61-79, 203-204.

3 Migrant Population Division of National Health and Family Planning Commission of PRC: Report on China migrant population development in 2017. Beijing, China: China Population Press, 2017.

4 Li X X, Zhang H, Jiang S W, et al. Active Pulmonary tuberculosis case detection and treatment among floating population in China: An Effective Pilot. J Immigrant Minority Health, 2010; 12: $811-815$.

5 World Health Organization. Global tuberculosis report, 2017. WHO/HTM/TB/2017.23. Geneva, Switzerland: WHO, 2017.

6 Jiang S W, Liu X Q. Progress and prospect of tuberculosis control for the floating population in China. Chin J Antituberc 2014; 36: 798-801.

7 China Tuberculosis Control Collaboration. The effect of tuberculosis control in China. Lancet $2004 ; 364: 417-22$

8 Long Q, Li Y, Wang Y, et al. Barriers to accessing TB diagnosis for rural-to-urban migrants with chronic cough in Chongqing, China: A mixed methods study. BMC Health Serv Res 2008; 8: 202 .

9 Bele S, Jiang W, Lu H, et al. Population aging and migrant workers: bottlenecks in tuberculosis control in rural China. PLOS ONE 2014; 9: e88290.

10 Zhu M M, Wang J, Dib H H, Wang Z Z. Enhancing the management of cross-regional transfer of floating Tuberculosis cases by active follow-up and communication. Eur J Public Health $2011 ; 22: 577-582$

11 Zhao M G, Guan H Y, Yang Y Z. Analysis on the effect of management of tuberculosis patients in cross-regional floating population in Shenzhen. Chin J Antituberc 2010; 32: 598-599. 
12 Zhou Y, Zhu L G, Zhu Y F, et al. Analysis of the implementation status of cross-regional management procedures and treatment outcome of the trans-provincial floating of TB cases. Chin J Dis Control Prev 2012; 16: 874-876.

13 Liu S L, Shi Y H, Shen Y, He H, Zeng Q Q, Chang C. Analysis of the medical intention of TB and its influencing factors among floating population in six provinces in China. Chin $\mathrm{J}$ Antituberc 2018; 40: 296-301.

14 Li X, Jiang S W, Gao Y X, Liu X Q. Situation analysis on registration and treatment of migrant tuberculosis patients at tuberculosis dispensaries which initially confirmed diagnosis in Bering. Chin J Antituberc 2017; 39: 86-90.

15 Wei X, Chen J, Chen P, et al. Barriers to TB care for rural-to-urban migrant TB patients in Shanghai: a qualitative study. Trop Med Int Health 2009; 14: 754-760.

16 Li X Q, Li T G, Tan S Y. Males, ages $\geqslant 45$ years, businessperson, floating population, and rural residents may be considered high-risk groups for tuberculosis infection in Guangzhou, china: a review of 136,394 TB confirmed cases. Rev. Inst. Med. Trop. Sao Paulo 2013; 55: 366-368.

17 Guan H Y, Tan W G, Yang Y Z, Wu Q F, Che X L, Lv D L. Analysis on the effect of TB comprehensive management model among cross-regional floating population in Shenzhen city. Chin J Antituberc 2015; 37: 498-503.

18 Liu W P, Men K, Yang P R, Zhang T H. Anaylsis on epidemiology of tuberculosis patients in inter-regional floating population of Shaanxi Province (2009-2013). J of Pub Health and Prev Med 2015; 26: 10-12.

19 National Technic Steering Group of the Epidemiological Sampling Survey for Tuberculosis. Report on fourth national epidemiological Sampling Survey for Tuberculosis. Chin J Tuberc Respir Dis 2002; 25: 3-7.

20 Lawn S D, Bekker L G, Middelkoop K, Myer L, Wood R. Impact of HIV infection on the epidemiology of tuberculosis in a peri-urban community in South Africa: the need for agespecific interventions. Clin Infect Dis 2006; 42: 1040-1047.

21 Lin D Z, Luo Y Q, Li Y M, Zhang J A, Zeng J C, Xu J F. Epidemiological comparison of tuberculosis between local and floating population in Dongguan from 2008 to 2012. J Clin Intern Med 2014; 31: 385-387. 
22 Ma J J, Yuan Y L, Zhang T J, Gao Y, Wang Y L. Epidemiological characteristics of tuberculosis patients and clinic vising among floating population in Jilin province. Chin J of Public Health Eng 2018; 17: 29-32.

23 Floyd K, Glaziou P, Houben R M G J, Sumner T, White R G, Raviglione M. Global tuberculosis targets and milestones set for 2016-2035: definition and rationale. Int J Tuberc Lung Dis 2018; 22: $723-730$.

24 Zhu, L, Yang Y Z, Guan H Y, et al. Trends in drug-resistant tuberculosis after the implementation of the DOTS strategy in Shenzhen, China, 2000-2013. Int J Tuberc Lung Dis 2018; 21: 759-765.

25 Zhang Y, Ye J J, Yang C F, et al. Analysis of the cross-regional management and treatment effect of tuberculosis patients in floating population of Hubei Province. Chin J Antituberc 2016; 38: $405-410$

26 Lin S F, Dai Z S, Wei S Z, Du Y C. Effectiveness of treatment and management of pulmonary tuberculosis cases among cross -regional floating population. Chin Tropical Med 2011; 11: $1334-1335,1370$.

27 Yu W B, Liu R G. Analysis on the influence of health administrative intervention on the referral of tuberculosis patients. J of Pub Health and Prev Med 2012; 23: 128-129.

28 Jia Z W, Jia X W, Liu Y X, et al. Spatial analysis of tuberculosis cases in migrants and permanent residents, Beijing, 2000-2006. Emerging Infectious Diseases 2008; 14: 1413-1419.

29 Dowd J B, Fletcher H A, Boccia D. Social determinants and BCG efficacy: a call for a sociobiological approach to TB prevention. F1000Research 2018; 7: 224.

30 Castro K G, Colvin C E. Updated global tuberculosis targets: a welcome ambition in need of attention to quality of care. Int J Tuberc Lung Dis 2018; 22: 709.

31 Lei F, Liu K W, Xu L, Li J W. The spatiotemporal characteristics and influencing factors of floating population in Shaanxi province: A study on the fourth, fifth and sixth census. Journal of Shaanxi Normal University (Natural Science Edition) 2015;43(2):92-98. 


\section{Figure legend:}

1. Table 1. Socio-demographic characteristics of TB cases

2. Table 2. Sex distribution of TB cases in inter-regional floating Population from 2009 to 2017

3. Table 3. Sex distribution of TB cases in inter-regional floating Population in different age group

4. Table 4. Inspection and treatment of TB cases in inter-regional floating Population

5. Table 5. Management of TB cases in inter-regional floating Population

6. Figure 1. TB case number change in inter-regional floating Population from 2009 to 2017 
Figures

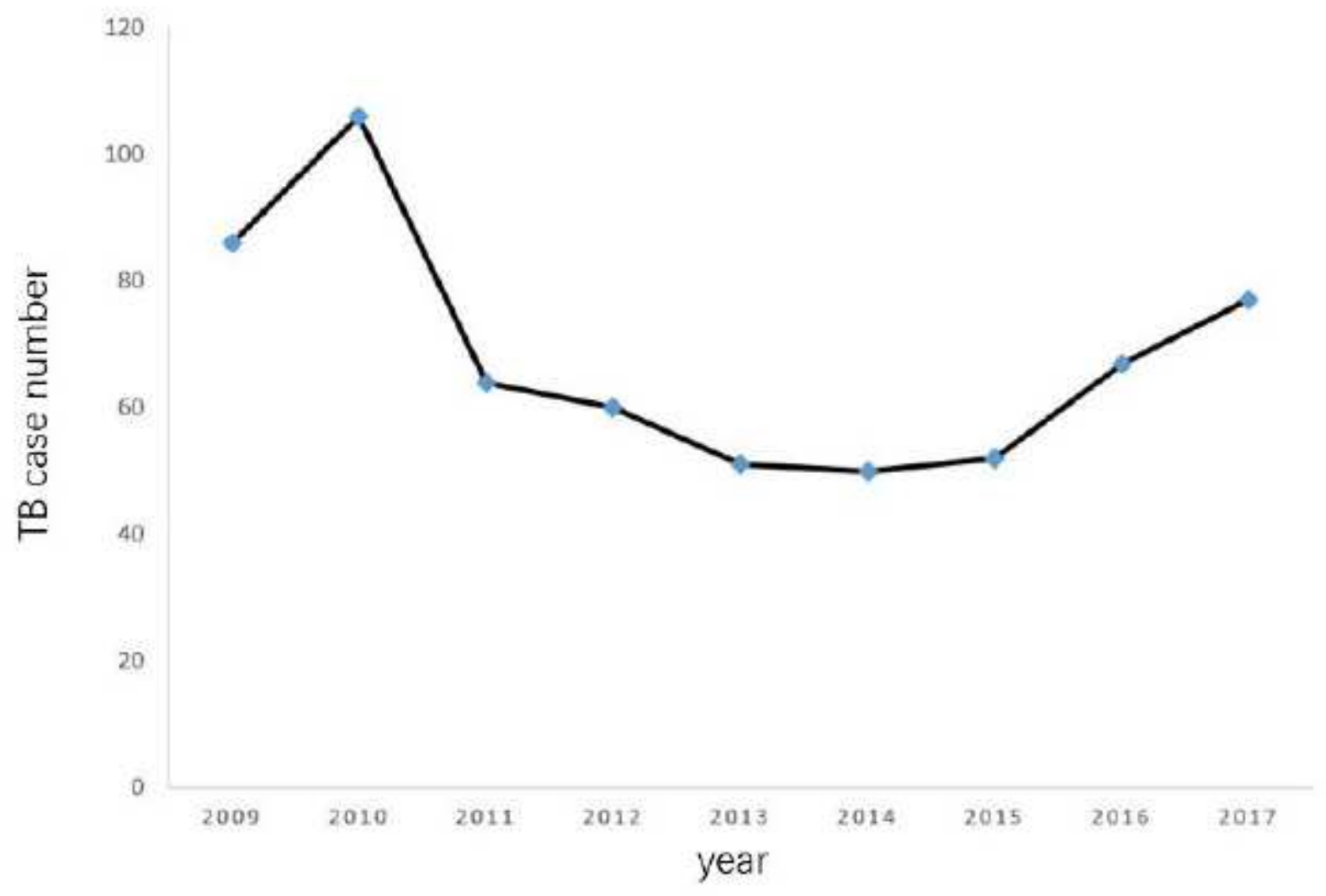

Figure 2

Figure 1 TB case number change in inter-regional floating Population from 2009 to 2017

\section{Supplementary Files}

This is a list of supplementary files associated with this preprint. Click to download.

- Table5.pdf

- Table3.pdf

- Table4.pdf

- Table5.pdf

- Table3.pdf

- Table4.pdf

- Table2.pdf

- Table1SociodemographiccharacteristicsofTBcases.pdf 
- Table1SociodemographiccharacteristicsofTBcases.pdf

- Table2.pdf 